\title{
Indian Society of Neuroanaesthesiology and Critical Care (ISNACC) Position Statement and Advisory for the Practice of Neuroanesthesia during COVID-19 Pandemic
}

\section{Endorsed by Indian Society of Anaesthesiologists (ISA)}

\author{
Kiran Jangra ${ }^{1, \odot ~ N i t i n ~ M a n o h a r ~}{ }^{2}$ Prasanna U. Bidkar ${ }^{3}$ \\ Joseph Monteiro ${ }^{7}$ Nidhi Panda ${ }^{1} \quad$ Kamath Sriganesh ${ }^{8}$

\footnotetext{
${ }^{1}$ Division of Neuroanaesthesia, Department of Anaesthesia and Intensive Care, Postgraduate Institute of Medical Education and Research, Chandigarh, India

2Department of Neuroanaesthesia and Neurocritical Care, Yashoda Hospitals, Secunderabad, Telangana, India

${ }^{3}$ Division of Neuroanesthesiology, Department of Anaesthesiology and Critical Care, Jawaharlal Institute of Postgraduate Medical Education and Research, Puducherry, India

${ }^{4}$ Department of Neuroanesthesiology and Neurocritical Care, MGM Health Care Pvt Ltd, Chennai, Tamil Nadu, India

${ }^{5}$ Department of Anesthesiology and Critical Care, Sanjay Gandhi Postgraduate Institute of Medical Sciences, Lucknow,

Uttar Pradesh, India

${ }^{6}$ Department of Neuroanaesthesiology and Critical Care, All India Institute of Medical Sciences, New Delhi, India

${ }^{7}$ Department of Anaesthesiology, P D Hinduja Hospital and Medical Research Center, Mumbai, Maharashtra, India
}

Ponniah Vanamoorthy ${ }^{4}$ Devendra Gupta ${ }^{5}$ Girija P. Rath ${ }^{6}$ (๑

Ajay P. Hrishi ${ }^{9} \quad$ Bhibukalyani Das $^{10}$ Rahul Yadav ${ }^{11}$

\begin{abstract}
Address for correspondence Prasanna Bidkar, MBBS, MD, DNB, DM, Division of Neuroanesthesiology, Department of Anaesthesiology and Critical Care, Jawaharlal Institute of Postgraduate Medical Education and Research, Puducherry 605006, India (e-mail: drprasannabidkar@gmail.com).
${ }^{8}$ Department of Neuroanaesthesiology and Neurocritical Care, National Institute of Mental Health Neurosciences, Bengaluru, Karnataka, India
${ }^{9}$ Division of Neuroanesthesiology, Department of Anesthesiology, Sree Chitra Tirunal Institute of Medical Sciences and Technology, Thiruvanathapuram, India Kolkata, West Bengal, India India

${ }^{10}$ Department of Neuroanaesthesia, Institute of Neurosciences,

${ }^{11}$ Department of Anaesthesia, INHS Asvini, Mumbai, Maharashtra,
\end{abstract}

\begin{abstract}
Keywords

- COVID-19 pandemic

- neuroanesthesia practice

- emergency neurosurgery

- neurointervention

The coronavirus disease 2019 (COVID-19) is a major health emergency in today's time. In December 2019, a cluster of pneumonia cases in Wuhan, China was attributed to a novel coronavirus. The World Health Organization declared it as a pandemic. As the majority of the cases suffering from COVID-19 are mildly symptomatic or asymptomatic, it becomes a great challenge to identify the infected persons in the absence of extensive testing. In the hospital environment, it can infect several other vulnerable patients and healthcare providers, significantly impacting the hospital services. Anesthesiologists are at an increased risk of COVID-19 transmission from the patients, as they are frequently involved in several aerosol-generating procedures. It is not possible to identify asymptomatic COVID-19 patients solely based on history-taking during their first point of contact with the anesthesiologists at the preanesthetic checkup clinic.

Most of the neurosurgical conditions are of urgent in nature and cannot be postponed for a longer duration. In view of this, the position statement and practice advisory from the Indian Society of Neuroanaesthesiology and Critical Care (ISNACC) provides guidance to the practice of neuroanesthesia in the present scenario. The advisory has been prepared considering the current disease status of the COVID-19 pandemic, available literature, and consensus from experts in the field of neuroanesthesiology. Since the pandemic is still progressing and the nature of the disease is dynamic, readers are advised to constantly look for updated literature from ISNACC and other neurology and neurosurgical societies.
\end{abstract}

DOI https://doi.org/ 10.1055/s-0040-1714186 ISSN 2348-0548. (c) 2020. Indian Society of Neuroanaesthesiology and Critical Care. This is an open access article published by Thieme under the terms of the Creative Commons Attribution-NonDerivative-NonCommercial-License, permitting copying and reproduction so long as the original work is given appropriate credit. Contents may not be used for commercial purposes, or adapted, remixed, transformed or built upon. (https://creativecommons.org/licenses/by-nc-nd/4.0/).

Thieme Medical and Scientific Publishers Pvt. Ltd., A-12, 2nd Floor, Sector 2, Noida-201301 UP, India 


\section{Introduction}

Coronavirus disease (COVID-19) is a severe acute respiratory syndrome (SARS) caused by SARS CoV-2. It started from China and has now been declared as pandemic., ${ }^{1,2}$ In India, approximately 510,672 COVID-19 cases and 15,712 deaths have been reported as of June 27, 2020 and the number is on a steep rise. As majority of the cases suffering from COVID-19 are mildly symptomatic or asymptomatic, it becomes a great challenge to identify the infected patients in the absence of extensive testing. A neurologically ill patient who is asymptomatic for COVID-19 acts as a silent spreader and has the potential to infect several other vulnerable patients and healthcare providers (HCPs).

Anesthesiologists are at an increased risk of COVID-19 transmission from the patients as they are frequently involved in several aerosol-generating procedures (AGPs) such as bag-mask ventilation, high-flow oxygen delivery, tracheal intubation and extubation, noninvasive and invasive ventilation, suctioning of airway secretions, and cardiopulmonary resuscitation. Moreover, it is not possible to identify asymptomatic COVID-19 patients solely based on the history taking during their first point of contact with the anesthesiologists at the preanesthetic checkup (PAC) clinic. The closed environment of the operation theater (OT) also increases the risk of COVID-19 transmission among the anesthesiologists and other HCPs during surgery. In these settings, effective infection control measures become essential components of patient care to limit COVID-19 transmission. Similarly, the use of appropriate personal protective equipment (PPE) by the anesthesiologists and other HCPs is pivotal during the clinical care of patients to minimize transmission and optimize self-protection.

Several health agencies such as World Health Organization (WHO) and Indian Council of Medical Research (ICMR) are frequently updating their advisories based on emerging evidence, in order to keep HCPs abreast of the rapidly evolving situation. Most of the neurosurgical conditions are essential surgeries which cannot be postponed for a longer duration. There is not much evidence to guide the approach toward anesthesia practice for neurosurgery in India during the COVID-19 times. In view of this, the current position statement and practice advisory from the Indian Society of Neuroanaesthesiology and Critical Care (ISNACC) is aimed to provide updates and guidance to the neuroanesthesiologists for managing the patients in the present scenario. Further, the present document can be used for making local policies and practice parameters. With the understanding about the disease constantly evolving, the advisory will be updated as knowledge evolves on this subject.

\section{Methods}

The ISNACC Governing Council formed a team of expert neuroanesthesiologists from across the country in April 2020 to formulate this advisory for the practice of neuroanesthesia during the COVID-19 pandemic. A detailed search of PubMed, Embase, Medline, OVID, and Google scholar databases was performed to explore information on neuroanesthesia and COVID-19. The articles that were considered were original investigations, case series, and the articles published exclusively in the English language. The search included the following specific words: COVID-19 and elective or emergency neurosurgery, neurological diseases, craniotomy, spine surgery, brain tumors, endoscopic surgery, and anesthesia. Information was also obtained from the guidelines and practice advisories of the allied specialties such as neurology, neurosurgery, anesthesiology, and intensive care. Additional information was retrieved from the cross references of these articles.

The directives from the Ministry of Health and Family Welfare (MOHFW), Government of India and ICMR were obtained from their respective websites. Since definitive evidence was not available, the committee members reached out to other experts in the field of neuroanesthesiology from across India to obtain their views. Multiple web-based meetings were held to discuss the available materials and formulate this position statement and advisory. The final version was approved by all the committee members.

\section{Preanesthetic Assessment during COVID-19 Pandemic}

Once the need for surgery is determined, the neuroanesthesiology team responsible for the conduct of anesthesia or the anesthesiologist at the PAC clinic will be contacted by the surgical team for preoperative assessment as per the hospital policy. The attending neuroanesthesiology team will work closely with the neurosurgical team and the COVID-19 team of the hospital to determine the subsequent workflow and work out careful patient selection. The subsequent care pathway will depend on the nature of surgery (emergent, urgent, or elective), the COVID-19 status (positive, suspect or negative) and the severity of COVID-19 infection, as shown in - Fig. 1 .

\section{Patients Symptomatic for COVID-19}

All symptomatic patients are evaluated in a preidentified isolation area. Video conferencing or teleconsultation is preferred to decide on the timing of surgery. All symptomatic patients must be directed to the COVID-19 management and elective surgeries should be deferred until they recover from infection. Symptoms described as influenza-like illness (ILI) such as fever (temperature $>38^{\circ} \mathrm{C}$ ) and cough are typical manifestations of COVID19 , and it is recommended to isolate these patients in the screening area itself. Apart from these symptoms, patients may present with atypical manifestations such as myalgia, sore throat, alteration in taste or smell, gastrointestinal symptoms, and cardiovascular manifestations. Neurological manifestations of COVID-19 have also been reported. Hypoxemia without respiratory distress (happy hypoxemia) has been reported in COVID-19. In view of this, it is recommended to check peripheral oxygen saturation $\left(\mathrm{SpO}_{2}\right)$ of all the patients during PAC. Patients who are 


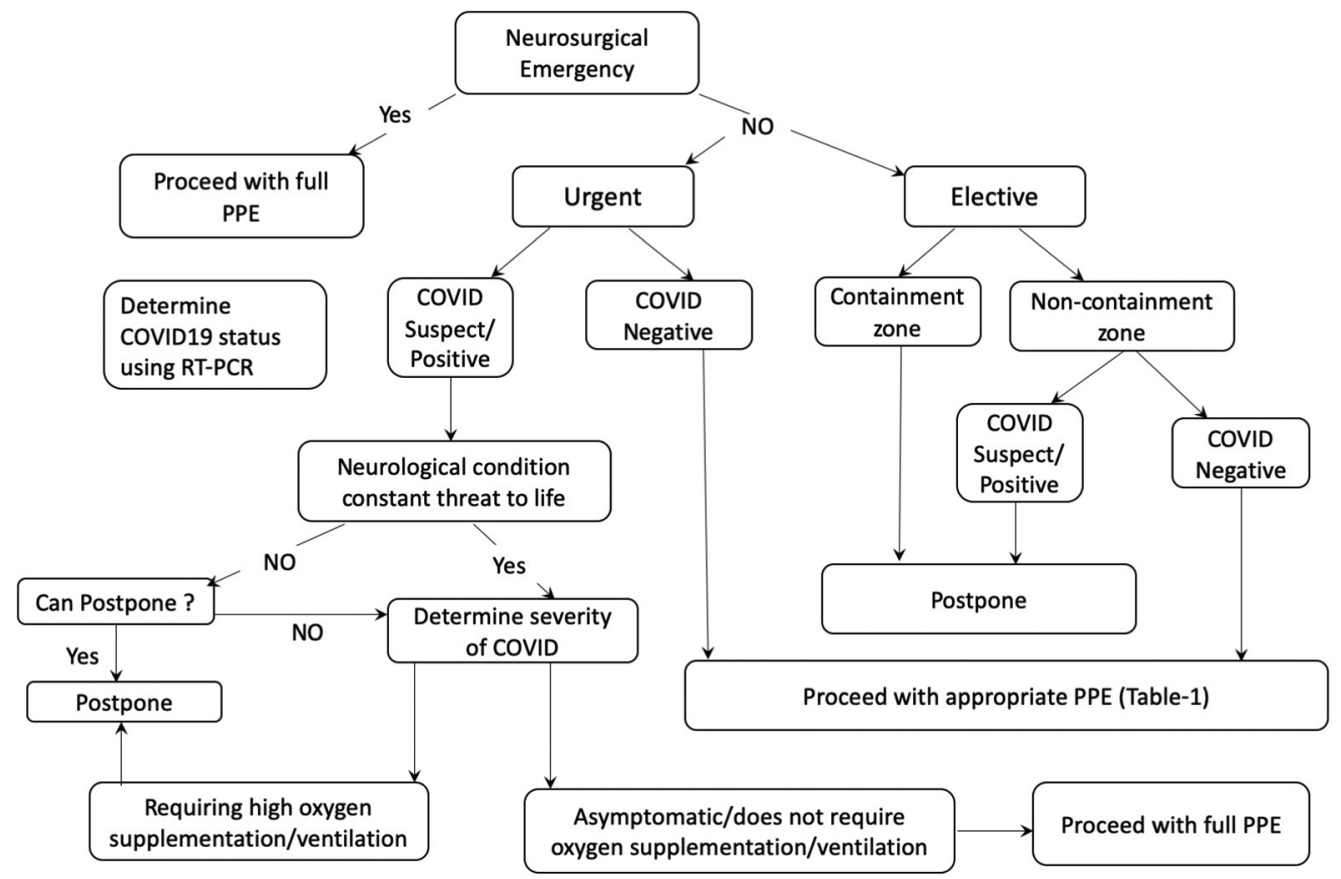

Fig. 1 Planning of neurosurgical patients presenting for surgery. COVID-19, corona virus disease-2019; PPE, personal protective equipment; RT-PCR, reverse transcription polymerase chain reaction.

severely hypoxemic $\left(\mathrm{SpO}_{2}<90 \%\right)$, despite oxygen therapy, should not be taken up for any surgery (including urgent and emergent) until the oxygenation improves. Till then, supportive treatment should be undertaken to manage raised intracranial pressure (ICP). ${ }^{3}$

\section{Patients Asymptomatic for COVID-19}

High-incidence of asymptomatic COVID-19 infection is reported in population studies. Therefore, it is imperative that HCPs including neuroanesthesiologists adhere to effective infection control precautions and use appropriate PPE during the clinical care of their patients. - Table 1 informs the MOHFW guidelines for the rational use of PPEs in nonCOVID-19 hospitals and non-COVID-19 treatment areas of any hospital. A high-index of suspicion and thorough workup is warranted in these patients.

\section{Preoperative Testing for COVID-19}

The current policy determined by the ICMR, MOHFW, and municipal health authorities in different states involves screening all the patients visiting the hospital and the accompanying person for symptoms of COVID-19. ${ }^{4}$ This is done by way of noncontact temperature check and careful history-taking with regard to contact with a COVID-19 patient, recent travel from high-risk areas, and residence in containment zones, identified regularly by the authorities, apart from symptoms of ILI such as fever and cough. All patients fulfilling the above criteria should undergo nucleic acid amplification testing for COVID-19. Currently, COVID-19 testing of other patients is unclear and depends on the policies framed by individual hospitals or local authorities. However, given the high-morbidity and mortality in COVID-19 patients undergoing surgery, it is advisable to test all neurosurgical patients for COVID-19.4-6 This helps all stakeholders in the decision-making regarding proceeding with neurosurgery or continuing maximal medical management, especially in elderly patients with significant comorbidities and uncertain neurological outcomes. It should also be remembered that false negative results are high when testing is done early after the exposure or when the patient is still asymptomatic, which gives a false sense of security. If at any time during the hospital stay, patients develop symptoms of ILI or atypical symptoms of COVID-19, they should be subjected to nucleic acid amplification testing for COVID-19. If the test report of the patient is positive, asymptomatic direct contacts including HCPs should also be tested for COVID-19.

The nasal and throat swabs of all emergency neurosurgical patients fulfilling the above screening criteria should be obtained for nucleic acid amplification testing for COVID-19, and emergency neurosurgery should proceed with full PPE. These patients should be managed in a designated COVID-19 suspect area until their report is available. If the report is positive, they should be shifted to the COVID-19 area of the hospital or the designated COVID-19 hospital, and if negative, to the usual postoperative area in the hospital.

All patients fulfilling the screening criteria and requiring urgent or elective neurosurgery should undergo COVID19 testing before surgery. If tested negative, they should undergo surgery with HCPs, including neuroanesthesiologists, wearing appropriate PPE in non-COVID-19 hospital and non-COVID-19 treatment areas of the hospital as mentioned in - Table 1. All elective neurosurgical procedures 
Table 1 Guidelines for the use of PPE in non-COVID-19 areas $^{\text {a }}$

\begin{tabular}{|c|c|c|c|c|c|}
\hline SI. No. & Setting & Activity & Risk & Recommended PPE & Remarks \\
\hline 1. & Doctors chamber & Clinical management & Mild risk & $\begin{array}{l}\text { - Triple layer medical mask } \\
\text { - Latex examination gloves }\end{array}$ & $\begin{array}{l}\text { No aerosol-generating pro- } \\
\text { cedures should be allowed }\end{array}$ \\
\hline 2. & $\begin{array}{l}\text { Preanesthetic } \\
\text { checkup (PAC) clinic }\end{array}$ & $\begin{array}{l}\text { Preanesthetic } \\
\text { checkup }\end{array}$ & Moderate risk & $\begin{array}{l}\text { - } \mathrm{N}-95 \text { mask } \\
\text { - Goggles* } \\
\text { - Latex examination gloves }\end{array}$ & $\begin{array}{l}\text { * Only recommended when } \\
\text { close examination of oral } \\
\text { cavity/dentures is to be } \\
\text { done }\end{array}$ \\
\hline 3. & $\begin{array}{l}\text { Ward/individual } \\
\text { rooms }\end{array}$ & Clinical management & Mild risk & $\begin{array}{l}\text { - Triple layer medical mask } \\
\text { - Latex examination gloves }\end{array}$ & $\begin{array}{l}\text { Patients stable } \\
\text { No aerosol-generating } \\
\text { activity }\end{array}$ \\
\hline 4. & $\begin{array}{l}\text { Operation } \\
\text { theater (OT) }\end{array}$ & $\begin{array}{l}\text { Performing surgery, } \\
\text { administering general } \\
\text { anesthesia }\end{array}$ & Moderate risk & $\begin{array}{l}\text { - Triple layer medical mask } \\
\text { - Face shield (wherever } \\
\text { feasible) } \\
\text { - Sterile latex gloves } \\
\text { - + Goggles } \\
\text { - N-95 mask* }\end{array}$ & $\begin{array}{l}\text { Already OT staff shall be } \\
\text { wearing } \\
\text { For personnel involved } \\
\text { in aerosol-generating } \\
\text { procedures } \\
\text { *If the person being } \\
\text { operated upon is a resident } \\
\text { of containment zone }\end{array}$ \\
\hline 5. & Sanitation & $\begin{array}{l}\text { Cleaning frequently } \\
\text { touched surfaces/ } \\
\text { floor/changing linen }\end{array}$ & Low risk & $\begin{array}{l}\text { - Triple layer medical mask } \\
\text { - Latex examination gloves }\end{array}$ & \\
\hline 6. & Emergency & $\begin{array}{l}\text { Attending } \\
\text { emergency cases }\end{array}$ & Mild risk & $\begin{array}{l}\text { - Triple layer medical mask } \\
\text { - Latex examination gloves }\end{array}$ & $\begin{array}{l}\text { No aerosol-generating } \\
\text { procedures are } \\
\text { allowed }\end{array}$ \\
\hline 7. & & $\begin{array}{l}\text { Attending to } \\
\text { severely ill patients } \\
\text { while performing } \\
\text { aerosol-generating } \\
\text { procedure }\end{array}$ & High risk & $\begin{array}{l}\text { - Full complement of PPE } \\
\text { (N-95 mask, coverall, } \\
\text { - goggle, nitrile examination } \\
\text { - gloves, shoe cover) }\end{array}$ & \\
\hline
\end{tabular}

Abbreviation: PPE, personal protective equipment.

ahttps://www.mohfw.gov.in/pdf/UpdatedAdditionalguidelinesonrationaluseofPersonalProtectiveEquipmentsettingapproachforHealth functionariesworkinginnonCOVID19areas.pdf

should be postponed for COVID-19 positive patients until they become asymptomatic and are tested negative for COVID-19. A patient may remain infectious until fever subsides without the use of fever-reducing medications, or respiratory symptoms improve, or two SARS-CoV-2 test results $\geq 24$ hours apart are negative. All urgent neurosurgical procedures for COVID-19 positive patients should be postponed, if possible. If the postponement is not possible, surgery should be performed with full PPE.

\section{General Guidelines}

1. Apart from routine evaluation for anesthesia for neurosurgical procedures, preanesthetic evaluation should include patient's exercise capacity (metabolic equivalents or METS) assessment and $\mathrm{SpO}_{2}$. Wherever possible, teleconsultation should be considered for preoperative evaluation of these patients.

2. Appropriate PPE should be used in various areas as per the MOHFW guidelines (- Table 1) ${ }^{7}$

3. The aerosol spread in the OT should be minimized during AGPs by avoiding high-flow oxygen, nasal instrumentation, coughing, bag and mask ventilation, and by use of aerosol prevention boxes.

4. Ideally, intubation should be performed by an experienced anesthesiologist using a videolaryngoscope. All
AGPs should preferably be conducted in a negative-pressure room, if available. However, considering the lack of availability of such rooms, central air conditioning with high-efficiency particulate air (HEPA) filters with $>25$ fresh air exchanges per hour should be used.

5. All disposable items such as drapes, breathing circuit, heat and moisture exchanger with viral filter (HMEF), gas sampling tubing, reservoir bag, anatomical face mask and airways should be discarded, and all exposed surfaces such as anesthesia work station and patient monitors should be cleaned with disinfectant solutions at the end of surgery.

6. The anesthesiologist should maintain a distance of at least $2 \mathrm{~m}$ from the surgical site, especially during placement of burr hole and craniotomy, and during the use of bone drilling equipment.

7. Wherever feasible, monitoring of the surgical field should be considered, using a slave monitor, and close communication with the surgeons should be maintained.

8. During other AGPs such as bone drilling, and using Cavitron ultrasonic surgical aspirator (CUSA) and monopolar cautery, suction should be held close to the surgical field and continuous irrigation should be considered.

9. The Indian Society of Anaesthesiologists has provided the detailed guidelines for perioperative management of

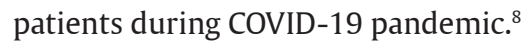




\section{Classification of Type of Surgeries}

\section{Emergency Neurosurgeries}

The emergency neurosurgeries include pathologies which are a constant threat to the life and limb (acute intracranial hypertension with deteriorating consciousness, rapidly developing hydrocephalus, and spinal cord compression with new-onset weakness).9,10 These need to be addressed as soon as possible. The emergency surgeries should not be delayed for lack of availability of test report. At places where testing facilities are not available, patients should be treated as COVID-19 suspect, and a nasopharyngeal swab sample should be taken prior to the shifting of the patients to the designated OT. Many times, appropriate history is also not available. In both these scenarios, full PPE should be used, and these patients should be considered as COVID-19 suspect/positive cases. Postoperatively, the patients should be transferred to and cared for in a predetermined isolated area using separate corridors till the test results are obtained.

\section{Urgent Neurosurgeries}

This category includes surgeries that are not immediately life-threatening (intracranial tumors with mass effect or with progressive neurological deficits), but may lead to severe neurological damage, if not treated..$^{9,10}$

\section{Spine Surgeries}

Spine conditions where pain and neurological manifestation can be reasonably managed nonoperatively are postponed routinely. Urgent spine surgeries are an appropriate option in patients where there is a risk of permanent but potentially reversible neurological dysfunction, and a pathology limiting the daily activities due to severe pain and deformity.

\section{Key Points for Spine Surgery}

Minimally invasive techniques when feasible and safe should be considered, as they decrease the risk of virus transmission from body fluids. Caution must be taken to avoid aerosol spread while turning the patient prone for surgery. It is advisable to minimize disconnection of the circuit during the change of position. If disconnection is mandatory, the tube should be clamped after full expiration, and the clamp should be removed only after reconnecting the circuit. Antisialagogues should be used to minimize drooling of saliva in prone position. Surgery should be performed by the most experienced neurosurgeon, and training of residents and fellows should take backstage during this period. Caution should be taken to avoid splattering of body fluids, and use of a smoke evacuator to minimize electrocautery smoke in the OT should be considered. ${ }^{11}$

For patients with unstable cervical spine, airway management should be performed by a senior anesthesiologist, and awake fiberoptic intubation (FOI) is better avoided as it can induce coughing despite maximal preventive efforts. ${ }^{12}$ The FOI can be performed under deep sedation or under general anesthesia (GA). Transtracheal blocks, local anesthetic viscous gargles, and nebulization are considered high AGPs, and they should be avoided. Alternatively, videolaryngoscope can be used to obtain a good laryngeal view with minimal neck movement. Awake prone positioning of the patient should be avoided as it can precipitate coughing.

\section{Neuro-oncologic Surgeries}

For brain tumor surgeries, the definitions of "emergent" and "elective" procedures are not well-demarcated, but it can be divided based on clinical and radiological factors. The neuro-oncology teams need to assess the risk and benefit of oncologic therapy during a pandemic. The patients should be triaged according to the urgency of management.

The decision of surgery versus conservative management is very complex. Surgery is usually needed to reduce mass effect of the tumor on the brain and spinal cord, and to retain and/or improve neurologic function. At many centers, all elective surgeries including for benign intracranial tumors with minor symptoms are currently deferred. ${ }^{12}$ For tumors involving lateral or third ventricle and pineal gland causing hydrocephalus, temporizing measures such as endoscopic third ventriculostomy or ventriculoperitoneal shunt may be considered. Definitive surgery may be delayed with a few exceptions, for example, germ cell tumors and pineoblastoma. ${ }^{13}$

\section{Aneurysmal Subarachnoid Hemorrhage}

A ruptured intracranial aneurysm is an urgent condition, as rebleed is a devastating complication, which is associated with a very high risk of morbidity and mortality. Rebleeding can be prevented by securing the aneurysm as early as possible. Most of the ruptured aneurysms are treated as an urgent procedure, except for patients who have signs of severely raised ICP with poor clinical grades. Such patients are managed with temporizing procedures such as external ventricular drain (until they improve clinically).

Treatment of unruptured aneurysm is considered as an elective procedure and should be postponed including for giant aneurysm till the patient is negative for COVID-19. The only exception would be patients presenting with new third nerve palsies. All patients should undergo computed tomography angiography (CTA) prior to transfer to a higher center with neurosurgical facilities. ${ }^{12}$ If an aneurysm is diagnosed, the clinical grade of SAH and risk factors affecting the outcome are assessed. Patients with good grades of SAH-World Federation of Neurosurgical Societies (WFNS) 1-3 should be managed as per the existing treatment guidelines. Patients with poor grades (WFNS 4-5) should be reassessed further, depending upon other prognostic factors, to know if patients will benefit from neurosurgical treatment. ${ }^{14}$ The poor prognostic factors include extreme age, pre-existing severe comorbidities, and major end-organ dysfunction. These patients may be managed conservatively in their local hospital until their general condition improves. 


\section{Special Situations}

\section{Transnasal and Transoral Surgeries}

All surgeries (endoscopic, microscopic and open) involving the nasal cavities are considered as high risk as nasal and oral cavities are reservoirs of high viral load. ${ }^{15}$ However, performing all pituitary surgeries using a transcranial approach during the pandemic also seems unnecessary. ${ }^{3}$ It is advisable to postpone these surgeries to a later date unless tumours are malignant or there is neurological deterioration such as worsening of vision or pituitary apoplexy. ${ }^{16}$

Preoperative preparation should include povidoneiodine nasal irrigation and oral rinse to reduce the potential viral load. ${ }^{17,18}$ During endoscopic transnasal surgery, highspeed drills should be avoided, as they promote aerosolization of potentially infected mucous particles. ${ }^{19}$ In some cases, transcranial approach should be considered instead of the transnasal approach, if additional morbidity is less likely. Alternatively, the minimally invasive sublabial microscopic approach can be used. The packing of the oropharyngeal cavity can be done using betadine-soaked ribbon gauze. ${ }^{17}$ Special precaution should be taken while removing these packs and suctioning of the oral cavity to avoid the generation of aerosols.

Transoral approach for surgeries, such as mechanical decompression of odontoid vicious callus, craniovertebral malformations, extirpation of $\mathrm{C} 1 / \mathrm{C} 2$, and surgery for inferior clivus tumors, is considered high-risk because of high-viral load in the pharyngeal mucosa and aerosol generation during surgery. Mouthwash with povidone-iodine prior to intubation can be considered to decrease the viral load. ${ }^{19}$

\section{Intraoperative Neuromonitoring}

Intraoperative neuromonitoring (IONM) should be used rationally in consultation with the neurosurgical team. The IONM personnel should be trained about PPE usage and social distancing. It is recommended to minimize IONM staff inside the OT and consider remote monitoring wherever feasible. Antiseptic wipes should be used to clean all surfaces of the IONM equipment that has entered the OT. Transparent plastic bags may be used to cover IONM equipment in COVID-19 positive or suspect patients, which can be cleaned with $70 \%$ alcohol (disinfecting solution/antiseptic wipe) after the procedure. ${ }^{20}$ This minimizes equipment contamination and the amount of time the IONM technician needs to stay in the room. Disposable electrodes should be utilized whenever possible. If the use of disposable electrodes is not possible, then the used needle electrodes should be cleaned with $70 \%$ alcohol wipes for 30 seconds, followed by ethylene oxide sterilization at 51 to $56^{\circ} \mathrm{C}$.

\section{Awake Craniotomy}

Awake craniotomy is preferred in patients where the tumor lies close to eloquent areas of the brain or during epilepsy surgeries to precisely locate the focus. ${ }^{21,22}$ Currently, literature is lacking regarding performing awake craniotomies during the COVID-19 pandemic. In most of the centers, awake craniotomies are not performed. However, for the patients in whom tumor is causing significant mass effect on eloquent areas and poses constant threat of significant disability, these surgeries can be offered. ${ }^{22}$

Awake craniotomy should be deferred in COVID-19 positive patients and in patients with respiratory symptoms due to any cause till they become asymptomatic and negative for COVID-19. A patient with an active chest infection is more likely to cough during the procedure, which can lead to brain bulge, resulting in difficulty in resection. Similarly, coughing may cause significant movement that may result in damage to the eloquent area during surgery. Finally, intraoperative coughing results in aerosol generation. If surgery is mandatory in such patients (e.g., tumor causing motor weakness), the surgery may be planned under GA with IONM. All measures should be taken to minimize the spread of aerosols, such as avoiding the high-flow of oxygen through a nasal cannula, insertion of the nasopharyngeal airway, oxygen delivery under the mask, and unnecessary airway interventions which can provoke cough. ${ }^{23}$ Patient's face should be covered with a surgical/N-95 mask to decrease the spread of aerosols.

Awake craniotomy is done using various anesthetic techniques including awake-awake-awake, asleep-awake, and asleep-awake-asleep. ${ }^{24}$ The anesthesiologist should choose a technique with which he/she is most comfortable. For asleepawake and asleep-awake-asleep techniques, airway instrumentation is needed, which may lead to production of aerosols. Hence, awake throughout technique with minimal sedation appears to be more suitable during the COVID-19 pandemic. ${ }^{24}$

An experienced anesthesiologist should perform scalp block to ensure higher success rate and adequate analgesia. Maintaining adequate distance during testing of speech and motor function is desirable for self-protection. Complete exposure of patient's face and adequate lighting is essential to monitor the response to testing from a distance.

\section{Pediatric Neurosurgery}

Similar to adult neurosurgery, pediatric neurosurgical procedures are also classified as follows: Emergency-death or disability is likely if surgery is deferred (e.g., surgery for significant mass effect from acute trauma or intracranial bleed, ruptured myelomeningocele, spinal cord compression, and acute hydrocephalus including due to shunt malfunction); Urgent-time sensitive (e.g., surgery for progressively symptomatic tumor, especially posterior fossa tumor); and Elective-that can safely be postponed to more than a month (e.g., surgery for craniofacial anomalies, Chiari malformation, chronic pain or spasticity needing baclofen pump placement or rhizotomy, and tethered cord). ${ }^{25}$

Neuroanesthesiologists should consider the routine use of preprocedural sedatives to reduce anxiety and increase compliance when an IV access is required in an awake child. Struggle during IV access placement may result in higher exposure to respiratory droplets from a crying child. Additionally, premedication may reduce risk of vigorous 
crying and agitation and the need for physical restraints during inhalational induction. Nasal administration of premedication is undesirable because of the potential for high-viral loads, and risk of coughing and sneezing. IV induction is preferred since inhalational induction may increase exposure to respiratory droplets and aerosols. Cuffed endotracheal tubes such as microcuff tubes should be preferred. Rapid sequence induction is recommended. If inhalational induction is chosen, it should be done with the lowest possible flow rate and maintenance of a tight mask seal. ${ }^{26}$

\section{Deep Brain Stimulation}

These surgeries are aimed to improve symptoms and quality of life of patients having chronic neurologic disorders such as Parkinson's disease. As these patients suffer from chronic illness, are often elderly and have several comorbidities, and they are more vulnerable to COVID-19 disease. These patients require surgery for placement of a deep brain stimulation (DBS) device. This surgery is considered an elective procedure and is usually not performed during the COVID-19 pandemic. ${ }^{27}$

These patients may also present with various issues in the hardware which may need an urgent intervention, such as internal pulse generator (IPG) depletion, malfunctioning, or infection. Most of these surgeries are preferably done under local anesthesia (LA). All precautions to reduce aerosol burden and transmission of infection should be undertaken. Under normal circumstances, battery placement is done under GA; however, during the COVID-19 pandemic, it is advisable to do this procedure under LA with or without sedation.

\section{Anesthesia at Remote Locations Magnetic Resonance Imaging}

All patients should be screened for COVID-19 and severely hypoxemic patients should not undergo MRI until they recover. All elective diagnostic imaging procedures should be postponed. Wherever possible, GA should be avoided in the MRI suite. If needed, AGPs such as intubation and extubation should be planned in a separate room (preferably negative pressure room) or in a designated area with appropriate PPE. A cuffed endotracheal tube is preferred in all the patients and supraglottic devices should be avoided.

Standard cleaning procedure includes sanitization of imaging suite between the patients using quaternary ammonium, alcohol, or other environmental protection agency-approved disinfectants. ${ }^{28}$ The American College of Radiology recommends 60 minutes of downtime between the patients and after all surfaces have been thoroughly cleaned. Large stationary HEPA filters (except in MRI) in imaging suites allows for sedimentation and removal of aerosol particles.

\section{Neurointervention}

Incidence of acute ischemic stroke (AIS) is found to be higher among patients with severe COVID-19, requiring intensive care unit admission, and some of these patients might require mechanical thrombectomy (MT) in the intervention neuroradiology suite (INR).. ${ }^{29-33}$ Proper history may not be possible in patients with AIS due to neurologic impairment. It is recommended that all suspected patients undergo COVID-19 testing after MT, if it is not possible at the time of admission.

During this COVID-19 pandemic, GA versus monitored anesthesia care (MAC) should be considered on a caseto-case basis depending on patient condition, procedure requirement and institutional protocol. The conversion from MAC to GA should be carefully considered to avoid risks of emergency airway management. Prophylactic intubation should be considered in patients with dominant hemisphere and posterior circulation occlusions, very high National Institutes of Health Stroke Scale (NIHSS) score, poor Glasgow coma scale (GCS) score, and those with respiratory difficulty. The risk of transmission of infection with emergency intubation during the procedure is high.

If GA is considered, rapid sequence intubation should be performed using videolaryngoscope by an experienced anesthesiologist in a negative pressure room, if available, or in a designated area for intubation and extubation. Unnecessary or accidental disconnections of breathing circuits during transport should be minimized. While performing the procedure under MAC, covering the nose and mouth with a surgical mask over the nasal prongs or under the face mask should be considered. Inadvertent hypo/ hyperventilation should be avoided by using capnography. During MAC, conscious sedation with low-flow oxygen to achieve $\mathrm{SpO} 2>94 \%$ is desirable.

It is desirable to designate a separate COVID-19 INR suite for MT in centers with multiple INR suites. Effective cleaning of angiography equipment and suites will have an impact on turnover times and readiness for subsequent cases.

A CT or MR angiography can be performed for the diagnosis of ruptured cerebral aneurysms. Diagnostic cerebral angiography can be avoided unless it is likely to provide additional information or alter the management. Aneurysm coiling and embolization of arteriovenous malformations could be managed similarly with all due precautions after COVID-19 testing.

\section{Electroconvulsive Therapy}

Routinely, ECT is not considered as an elective procedure in patients with psychiatric illness. ${ }^{34}$ The decision of performing electroconvulsive therapy (ECT) is based on the severity of psychiatric illness. Patients receiving ECT typically require three treatment sessions per week, frequently for 2 or more weeks. Therefore, one-time testing for COVID-19 prior to treatment is not sufficient. Asymptomatic infected patients may become symptomatic, and noninfected patients may develop an infection during the repeated visits for ECT. Retesting of patients via nasopharyngeal swab before every ECT is also not feasible due to lengthy turnaround time. Hence, appropriate PPE should be worn for all ECTs during the COVID-19 pandemic. However, the patient should be 
screened for COVID-19 symptoms every time before anesthesia to plan further management.

If any patient is febrile or has symptoms suggestive of infection, ECT should be deferred until a nasal swab is obtained and confirmed to be negative. Anticholinergics should be considered to reduce secretions and their aerosolization. Suctioning of secretions can induce coughing and aerosolize the virus. Hyperventilation should be used with caution for reducing seizure threshold. Appropriate safety measures are therefore desirable.

\section{Resuming Elective Neurosurgery}

Readiness to return of planned elective surgery will vary across states, districts, and institutions, depending on variables such as space, staff, equipment, and systems available. Once started, it is likely that there will be a pressing demand to cater to a long-pending waiting list of patients in whom elective surgery was postponed due to the pandemic. All patients should undergo screening and testing as per recommended guidelines updated regularly. For chronic neurosurgical problems, conservative treatment should be considered in suspected or confirmed COVID-19 patients. ${ }^{35}$ Except for life, limb or sight saving procedures, elective neurosurgical procedures should be avoided during the pandemic, as their outcomes may be complicated by increased morbidity and mortality. ${ }^{4}$ These patients may also pose a risk to attending HCPs and other vulnerable hospitalized patients. ${ }^{36}$

There should be a steady decline in new COVID-19 cases in concerned geographical area for $\geq 14$ days before the resumption of elective neurosurgeries, which should be congruent with the directives of Central/State Government, municipal authorities and institutional directives and protocols. Each institution has to form a select committee consisting of leaders from surgery, anesthesia, and nursing to prioritize and schedule procedures. It is important to identify capacity and realistic goals prior to undertaking resumption of elective neurosurgeries.

For elective neurosurgeries requiring GA, the patient is transported to the OT, wearing a 3-ply surgical mask, and the body is covered using a transparent disposable sheet. Since the risk of aerosol dispersion is maximal during airway instrumentation at the time of intubation and extubation, the entire face and neck should be covered with a transparent sheet on a metal prop, depending on local availability, and the edges should be secured safely to the operating table. This sheet can have either two linear or two cruciate incisions to snugly permit the anesthesiologist's access to the airway for intubation. At the end of the surgery, reversal and extubation are performed with the same precautions as intubation. There is no evidence to recommend the use of supraglottic devices at present. The presence of an induction room for intubation and extubation will further enhance the safety of healthcare personnel.

\section{Transportation to Imaging and Other Facilities}

Transportation of patients may be needed for performing brain and spine imaging or for special procedures conducted in a specific environment. For these reasons, patients may be transported within the hospital or outside to other centers. Infection control precautions should be taken during such transportations.

Unnecessary transport for investigations, which are not immediately needed, should be deferred. The entire team where the patient is to be transported should be notified regarding the COVID-19 status of the patient and the estimated time of arrival. The transportation team should wear appropriate PPE. If the patient is transported by the same team that is treating the patient, they should first doff and don again with new PPE before shifting the patient to other areas.

Special precautions should be taken as per the patient's condition. Spontaneously breathing patients should wear a surgical/N95 mask. In intubated patients, HMEF should be used to minimize the spread of aerosols. Patients may be covered with a transparent sheet during the transport. There should be a separate entry and exit pathways for COVID-19 positive patients. Patients should be transported through a separate corridor and this should not be shared by other patients or staff. If the transport corridor is shared, then that should be blocked for at least 30 minutes or till the sanitization is complete. Transporters should wear recommended PPE and eye protection. At the destination, the receiving personnel and the transport team should wear recommended PPE. Ambulance and transportation trolley should be sanitized at the end of the procedure.

\section{Conclusion}

At present, the COVID-19 pandemic is ongoing with more than 9.9 million people affected globally, with a steadily increasing death rate. With the surge in the number of patients and community spread in India, it appears difficult to perform elective neurosurgeries as in the pre-COVID-19 era. However, considering the urgent nature of most neurosurgical procedures, it is important to have a high-index of suspicion and perform laboratory testing for COVID-19. Appropriate PPE, as advised by the statutory bodies, should be used while anesthetizing these patients. Requirements of specific surgeries should also be kept in mind during perioperative management.

\section{Conflict of Interest}

G.P.R. serves as Director-at-Large of the Society for Neuroscience in Anesthesiology and Critical Care (SNACC) Board of Directors. 


\section{References}

1 Huang C, Wang Y, Li X, et al. Clinical features of patients infected with 2019 novel coronavirus in Wuhan, China. Lancet 2020;395(10223):497-506

2 World Health Organization. Coronavirus disease (COVID-19) Pandemic. Available at: https://www.who.int/emergencies/ diseases/novel-coronavirus-2019?gclid=Cj0KCQjwudb3BRC9ARIsAEa-vUsctFID6aobT3Kv3DULXTWG_n6djvGHw8YFr3DBVsdtI520KhEcsTwaAp8TEALw_wcB. Accessed April 9, 2020

3 Nahshon C, Bitterman A, Haddad R, Hazzan D, Lavie O. Hazardous postoperative outcomes of unexpected COVID-19 infected patients: a call for global consideration of sampling all asymptomatic patients before surgical treatment. World J Surg 2020. Doi: $10.1007 / \mathrm{s} 00268-020-05575-2$

4 Indian Council of Medical Research Department of Health Research, Ministry of Health and Family Welfare, Government of India. Advisory on Use of Rapid Antigen Detection Test for COVID-19. Published on: 14th June 2020. Available at: https:// www.icmr.gov.in/pdf/covid/strategy/Advisory_for_rapid_ antigen_test_14062020_.pdf. Accessed June 16, 2020

5 COVIDSurg Collaborative. Mortality and pulmonary complications in patients undergoing surgery with perioperative SARS-CoV-2 infection: an international cohort study. Lancet 2020.Doi: 10.1016/S0140-6736(20)31182-X

6 Tenenbein P, Riazi S, Johnstone J, Keshavjee S, Karkouti K. The case for routine screening for SARS-CoV-2 before surgery. Can J Anaesth 2020;3:1-6. Doi: 10.1007/s12630-020-01730-4

7 MOHFW. Ministry of Health and Family Welfare Directorate General of Health Services [Emergency Medical Relief]. Available at: https://www.mohfw.gov.in/pdf/Additionalguideline sonrationaluseofPersonalProtectiveEquipmentsettingapproachforHealthfunctionariesworkinginnonCOVIDareas.pdf. Accessed May 25, 2020

8 Malhotra N, Bajwa SJ, Joshi M, Mehdiratta L, Trikha A. COVID Operation Theatre- Advisory and Position Statement of Indian Society of Anaesthesiologists (ISA National). Indian J Anaesth 2020;64:355-362.

9 Jangra K, Paliwal S, Tripathi M. COVID-19 and neurosurgery: time for triage. J Neuroanaesth Crit Care 2020;07(2):067-069

10 Zoia C, Bongetta D, Veiceschi P, et al. Neurosurgery during the COVID-19 pandemic: update from Lombardy, northern Italy. Acta Neurochir (Wien) 2020;162(6):1221-1222

11 Lee Ting Soh T, Loong Ho SW, Quan Yap WM, Yoong-Leong Oh J. Spine surgery and COVID-19: challenges and strategies from the front lines. J Bone Joint Surg Am 2020. Doi: 10.2106/ JBJS.20.00503

12 Cook TM, El-Boghdadly K, McGuire B, McNarry AF, Patel A, Higgs $A$. Consensus guidelines for managing the airway in patients with COVID-19: Guidelines from the Difficult Airway Society, the Association of Anaesthetists the Intensive Care Society, the Faculty of Intensive Care Medicine and the Royal College of Anaesthetists. Anaesthesia 2020;75(6):785-799

13 Centers for Medicare \& Medicaid Services. Non-Emergent, Elective Medical Services, and Treatment Recommendations. Available at: https://www.cms.gov/files/document/cms-non-emergent-elective-medical-recommendations.pdf. Accessed May 21, 2020

14 The Society of British Neurological Surgeons. BNVG/SBNS Guide for the Neurosurgical Management of Neurovascular Conditions During the COVID-19 Pandemic. Available at: https://www.sbns.org.uk/index.php/policies-and-publications/covid/. Accessed May 21, 2020

15 Kolias A, Tysome J, Donnelly N, et al. A safe approach to surgery for pituitary and skull base lesions during the COVID-19 pandemic. Acta Neurochir (Wien) 2020;162(7):1509-1511
16 Huang $\mathrm{X}$, Zhu $\mathrm{W}$, Zhao $\mathrm{H}$, Jiang X. In reply: precautions for endoscopic transnasal skull base surgery during the COVID-19 pandemic. Neurosurgery 2020;17:nyaa145

17 Patel ZM, Fernandez-Miranda J, Hwang PH, et al. Letter: Precautions for Endoscopic Transnasal Skull Base Surgery During the COVID-19 Pandemic. Neurosurgery 2020;87(1):66-67

18 Mady LJ, Kubik MW, Baddour K, Snyderman CH, Rowan NR. Consideration of povidone-iodine as a public health intervention for COVID-19: Utilization as "Personal Protective Equipment" for frontline providers exposed in high-risk head and neck and skull base oncology care. Oral Oncol 2020;105:104724

19 Castelnuovo P, Turri-Zanoni M, Karligkiotis A. Skull base surgery during the Covid-19 pandemic. The Italian Skull Base Society Recommendations. Int Forum Allergy Rhinol 2020. Doi:10.1002/alr.22596

20 American Clinical Neurophysiological Society. Guidelines -Covid Resources. Available at: https://www.acns.org/practice/covid-19resources. Accessed May 22, 2020

21 Meng L, McDonagh DL, Berger MS, Gelb AW. Anesthesia for awake craniotomy: a how-to guide for the occasional practitioner. Can J Anaesth 2017;64(5):517-529

22 Meng L, Berger MS, Gelb AW. The potential benefits of awake craniotomy for brain tumor resection: an anesthesiologist's perspective. J Neurosurg Anesthesiol 2015;27(4):310-317

23 Simonds AK, Hanak A, Chatwin M, et al. Evaluation of droplet dispersion during non-invasive ventilation, oxygen therapy, nebuliser treatment and chest physiotherapy in clinical practice: implications for management of pandemic influenza and other airborne infections. Health Technol Assess 2010;14(46):131-172

24 Hansen E, Seemann M, Zech N, Doenitz C, Luerding R, Brawanski A. Awake craniotomies without any sedation: the awake-awake-awake technique. Acta Neurochir (Wien) 2013;155(8):1417-1424

25 Weiner HL, Adelson PD, Brockmeyer DL, et al. Editorial. Pediatric neurosurgery along with Children's Hospitals' innovations are rapid and uniform in response to the COVID-19 pandemic. J Neurosurg Pediatr 2020;1-3. Doi: 10.3171/2020.4.PEDS20240

26 Matava CT, Kovatsis PG, Summers JL, et al; PeDI-Collaborative. Pediatric airway management in COVID-19 patients - Consensus Guidelines from the Society for Pediatric Anesthesia's Pediatric Difficult Intubation Collaborative and the Canadian Pediatric Anesthesia Society. Anesth Analg 2020;10:1213

27 Emanuel EJ, Persad G, Upshur R, et al. Fair allocation of scarce medical resources in the time of covid-19. N Engl J Med 2020;382(21):2049-2055

28 Environmental Protection Agency. List N: Disinfectants for Use Against SARS-CoV-2 (COVID-19). Available at: https://www.epa. gov/pesticide-registration/list-n-disinfectants-use-against-sarscov-2-covid-19. Accessed June 18, 2020

29 Fraser JF, Arthur AS, Chen M, et al. Society of NeuroInterventional Surgery recommendations for the care of emergent neurointerventional patients in the setting of COVID-19. J Neurointerv Surg 2020;12(6):539-541

30 Sharma D, Rasmussen M, Han R, et al. Anesthetic management of endovascular treatment of acute Ischemic stroke during COVID-19 pandemic: consensus statement from society for neuroscience in anesthesiology \& critical care (SNACC) J Neurosurg Anesthesiol 2020;32(3):193-201

31 Flexman AM, Abcejo AS, Avitsian R, et al. Neuroanesthesia practice during the COVID-19 pandemic: recommendations from Society for Neuroscience in Anesthesiology and Critical Care (SNACC) J Neurosurg Anesthesiol 2020;32(3): 202-209 
32 Mao L, Jin H, Wang M, et al. Neurologic manifestations of hospitalized patients with Coronavirus disease 2019 in Wuhan, China. JAMA Neurol 2020;77(6):683-690

33 Oxley TJ, Mocco J, Majidi S, et al. Large-vessel stroke as a presenting feature of Covid-19 in the young. N Engl J Med 2020;382(20):e60

34 American Psychiatric Association. Practice Guidance for COVID-19. Available at: https://www.psychiatry.org/psychiatrists/covid-19-coronavirus/practice-guidance-for-covid-19. Accessed June 18, 2020
35 Gupta P, Muthukumar N, Rajshekhar V, et al. Neurosurgery and neurology practices during the novel COVID-19 Pandemic: A consensus statement from India. Neurol India 2020;68(2): 246-254

36 American Society of Anesthesiologists. Joint Statement: Roadmap for Resuming Elective surgery after Covid19Pandemic. Available at: https://www.asahq.org/about-asa/newsroom/news-releases/ 2020/04/joint-statement-on-elective-surgery-after-covid-19pandemic. Accessed June 18, 2020 